\title{
A broadband, high common-mode rejection ratio instrumentation amplifier
}

\author{
Author(s) \\ van der Horst, Marcel J. \\ DOl
}

10.1109/APEMC49932.2021.9596926

\section{Publication date}

2021

\section{Document Version}

Final published version

\section{License}

Unspecified

Link to publication

\section{Citation for published version (APA):}

van der Horst, M. J. (2021). A broadband, high common-mode

rejection ratio instrumentation amplifier. Paper presented at 2021 ASIA PACIFIC INTERNATIONAL SYMPOSIUM ON ELECTROMAGNETIC COMPATIBILITY, Kuta Selatan, Indonesia.

https://doi.org/10.1109/APEMC49932.2021.9596926

It is not permitted to download or to forward/distribute the text or part of it without the consent of the author(s) and/or copyright holder(s), other than for strictly personal, individual use, unless the work is under an open content license (like Creative Commons).

If you believe that digital publication of certain material infringes any of your rights or (privacy) interests,

please let the Library know, stating your reasons. In case of a legitimate complaint, the Library will make the material inaccessible and/or remove it from the website. Please contact the library:

https://www.amsterdamuas.com/library/contact/questions, or send a letter to: University Library (Library of the University of Amsterdam and Amsterdam University of Applied Sciences), Secretariat, Singel 425, 1012 WP Amsterdam, The Netherlands. You will be contacted as soon as possible. 


\title{
A Broadband, High Common-Mode Rejection Ratio Instrumentation Amplifier
}

\author{
Marcel J. van der Horst \\ Department of Electrical Engineering/ Amsterdam Sensor Lab \\ Amsterdam University of Applied Sciences, The Netherlands, m.j.van.der.horst@hva.nl
}

\begin{abstract}
An operational amplifier based instrumentation amplifier (IA) with a common-mode rejection ratio (CMRR) independent of resistance tolerances is presented in this paper. The CMRR is determined by the operational amplifier characteristics. The IA shows a high CMRR up to $100 \mathrm{kHz}$. Moreover, since the presented IA operates in the current domain, no large internal voltage swings occur, making it an interesting choice for low-voltage applications in situations where common-mode disturbances may affect the signal processing.

Index Terms-Nullor, instrumentation amplifier, common-mode rejection ratio, operational amplifier, common-mode disturbance
\end{abstract}

\section{INTRODUCTION}

Instrumentation amplifiers (IA) are typically used when common-mode (CM) signals may disturb the differential-mode (DM) signal transfer. Its task is to suppress the CM disturbance and amplify the DM signal. This ability is the common-mode rejection ratio. The $\mathrm{CM}$-signal may be at $\mathrm{DC}$, but is usually an AC signal. An example of the latter possibility is the measurement of bio-potentials like electrocardiogram and electroencephalogram where the mains may induce $\mathrm{CM}$ voltages on the order of several volts, while the signal of interest is in the order of (hundreds of) $\mu \mathrm{V}$ to several $\mathrm{mV}$ [1].

In general, increasing $\mathrm{CM}$ disturbance in the low-frequency $(2-150 \mathrm{kHz})$ range can be expected nowadays [2], increasing the demand for IAs with high (enough) CMRR in this band. The current trend towards low supply voltages requires the IA to meet this demand at decreasing supply voltages as well.

A lot of investigation has been performed over the years to improve the CMRR of IAs, e.g., [3][7]. Most focusing on integrated circuit IA design, which eases the design for a high CMRR.

Integrated circuit vendors supply various monolithic IAs based on e.g., current feedback, the wellknown three operational amplifier (op amp) IA or the two op amp IA. The CMRR is highly affected by the inequalities in resistor ratios in the subtractor in the latter two cases. In monolithic IAs these resistors are laser-trimmed, the transistors are well matched and can therefore provide better CMRRs than non-monolithic op amp based IAs [8]. IAs with high CMRR over a large frequency range and that are able to operate on low supply voltages are, however, still scarce.

This paper proposes an op amp based IA design with a CMRR that is not affected by inequalities of resistor(s) (ratios). Moreover, the CMRR is high over a broad frequency range and the IA can be used in low-voltage applications.

Section II presents the design of the IA. Gain and CMRR measurements are given in Section III and the design is discussed in Section IV. Finally, Section V gives the conclusions.

\section{INSTRUMENTATION AMPLIFIER DESIGN}

An IA should perform both an amplifying and a subtraction function to the signals at both of its inputs. This will result in cancellation of $\mathrm{CM}$ voltages and amplification of DM voltages. Figure 1 shows an effective subtraction function in the current domain. Nullors [9] are used to realize a

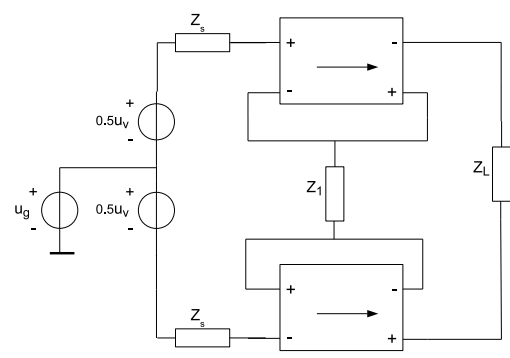

Fig. 1: Subtraction function: voltage in, current out.

balanced transadmittance amplifier.

The current through $Z_{L}$ is only determined by the DM voltage $u_{v}$. Due to the negative feedback action, the voltage difference across $Z_{1}$ caused by the CM voltage $u_{g}$ is zero. Therefore, no CM current will flow through $Z_{L}$. When $Z_{L}$ is formed by a (differential) transimpedance amplifier, an IA 
with balanced voltage output terminals is created; there are two output voltages with $180^{\circ}$ phase difference available. Here, an implementation using two unbalanced transimpedance amplifiers is used, see Fig.2.

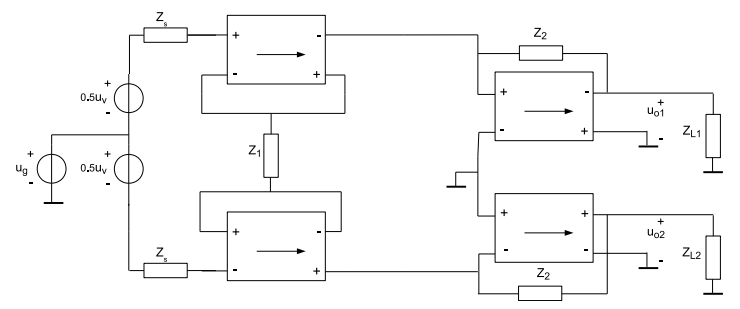

Fig. 2: IA with a balanced transadmittance and two transimpedance amplifiers. Using, e.g., a differential amplifier, one output voltage may be realized.

As a result of $u_{v}$, a current $-u_{v} / Z_{1}$ will start flowing in the output circuit. The transimpedance amplifiers convert this current into an output voltage according to $u_{o 1}=\left(u_{v} / Z_{1}\right) \cdot Z_{2}$ and $u_{o 2}=$ $-\left(u_{v} / Z_{1}\right) \cdot Z_{2}$. Hence, for the total voltage gain for both output follows

$$
\mu_{d}= \pm \frac{Z_{2}}{Z_{1}}
$$

\section{A. A nullor implementation with op amps}

Only in the case of the voltage amplifier and the transimpedance amplifier can the nullors be implemented with op amps without any problems. In case of the transadmittance amplifier, the problem is encountered that there is no differential, floating (isolated) output terminal pair. The problem to solve is to find a way of providing an isolated differential output pair using op amps only.

Huijsing presented an elegant solution based on two op amps and floating voltage sources [10]. Fig 3 shows his design. The maximum voltage swing is

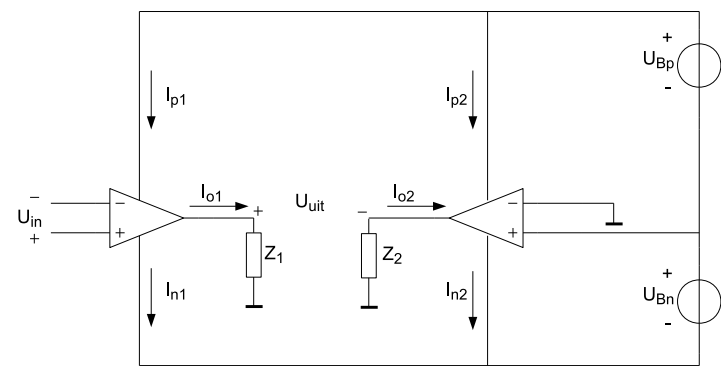

Fig. 3: A two opamp nullor implementation.

(almost) equal to $U_{B p}+U_{B n}$ when using op amps with rail-rail output.
The right op amp regulates its current $i_{o 2}$ such that the total positive and negative currents are equal to each other [10]. It holds that $i_{o 1}=$ $i_{p 1}-i_{n 1}, i_{o 2}=-i_{p 2}+i_{n 2}$ and $i_{t o t}=i_{p 1}+i_{p 2}=$ $i_{n 1}+i_{n 2}$, hence $i_{o 2}=i_{p 1}-i_{n 1}=i_{o 1}$ (when leakage currents from the op amps are ignored).

The floating supply voltage sources of the op amps must be isolated from the signal source and load reference: no galvanic contact and preferably negligible capacitance to that reference.

\section{B. CMRR limitations}

The CMRR that can be reached will be limited by both the performance of the input stage, i.e. the quality of the subtraction action, and the CMRR limitations of the op amps used (the same holds for the familiar 3 op amp IA). From a small-signal analysis follows that the CMRR in the former case can be approximated by

$$
\begin{aligned}
& C M R R(s) \approx \\
& 20 \cdot \log \left|\frac{1+2\left(A_{1}(s)+A_{2}(s)\right)+3 A_{1}(s) A_{2}(s)}{3\left(A_{1}(s)-A_{2}(s)\right)}\right| .
\end{aligned}
$$

$A_{1}(s)$ and $A_{2}(s)$ are the open loop gains of the op amps used and $s$ is the Laplace operator. The open loop gain and gain bandwidth product (GBP) are given in the datasheets, thus providing $A_{1}(s)$ and $A_{2}(s)$. Note that typical values are given that are prone to spread up to $30 \%$ [11].

It can be seen that for high CMRR values, $A_{1}(s)$ and $A_{2}(s)$ should be as equal as possible and/or as high as possible. When using commercially available op amps, selecting an op amp with high open loop gain is advised. Theoretically, CMRR $\gg 80 \mathrm{~dB}$ can be reached with high gain op amps. In that case the practically obtainable maximal CMRR will be determined by the op amp.

\section{IA Design}

Fig. 4 shows the design. The voltage gain $\mu_{d}$ is $40 \mathrm{~dB}$. A low value of only $10 \Omega$ was chosen for the feedback impedance $Z_{1}$ (Fig. 2) so its noise contribution is negligible. For $Z_{2}$ follows a resistor of $1 \mathrm{k} \Omega$. The amplifier is limited to a bandwidth of $15.9 \mathrm{kHz}$ with the capacitances shown in Fig. 4. The dual op amp AD822 is used. Ltspice simulations show a $\mu_{d}$ of $40 \mathrm{~dB}$ and an equivalent voltage noise of $18 \mathrm{nV} / \sqrt{\mathrm{Hz}}$ or $2.34 \mu \mathrm{V}_{r m s}$ total noise, determined by the input op amps. Op amp macromodels are not appropriate for CMRR simulations for this design, so simulation results are not given. Based on the op amp specifications and Eq. (2), calculations showed high CMRR up to $100 \mathrm{kHz}$. 


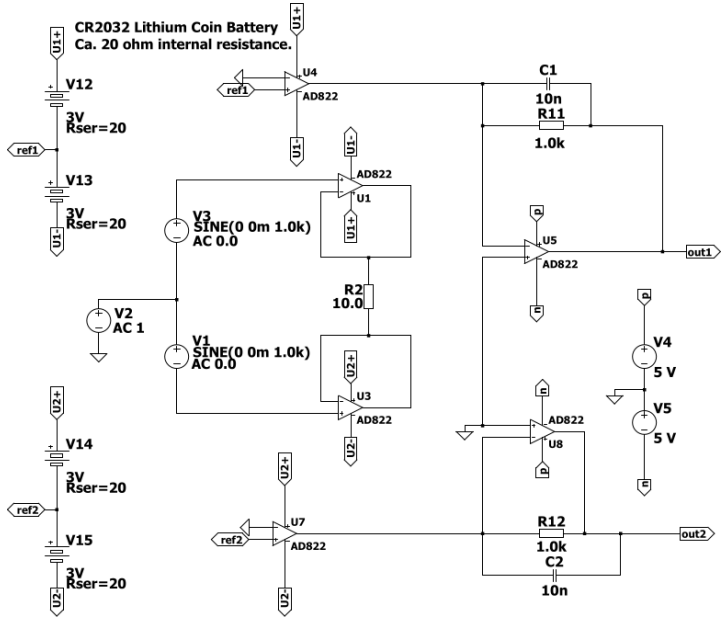

Fig. 4: The IA design.

In this design $3 \mathrm{~V}$ batteries are chosen as supply voltage of the input stage. The output stage is fed from $\pm 5 \mathrm{~V}$ derived from two $9 \mathrm{~V}$ batteries.

\section{Measurements}

The IA was realized on an experimental board and both $\mu_{v}$ and CM gain $\mu_{g}$ were measured using a Rigol DG1022 function generator and a Tektronix TBS 1072 oscilloscope. The differential voltage was set to $10 \mathrm{mV}_{\mathrm{rms}}$ and the resulting output voltage was measured over a frequency range of $10-100 \mathrm{kHz}$. See Fig. 5 for a picture of the IA.

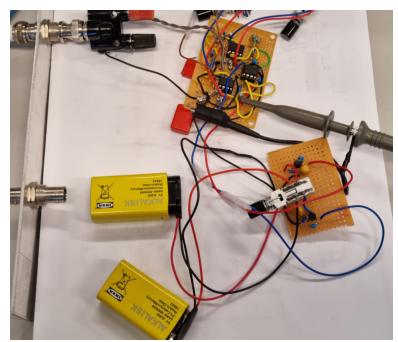

Fig. 5: The upper board shows three dual op amps forming the IA, the lower board shows the on-off switch and two voltage regulators $( \pm 5 \mathrm{~V})$.

$\mu_{g}$ was measured by connecting both inputs to the function generator and connecting the 'ground' side of it to the amplifier reference (see Fig. 2, with $u_{v}$ zero). The $\mathrm{CM}$ input voltage was $100 \mathrm{mV}_{\text {rms }}$. The CMRR was calculated with $20 \log \left(\mu_{d} / \mu_{g}\right)$.

To demonstrate the effect of $A_{1}(s)$ and $A_{2}(s)$ on the CMRR, measurements were performed with the AD 822 as input stage and the MCP6002, comparable CMRR but lower GBP, as input stage of the IA.
Fig. 6(a) shows the measured $\mu_{d}$ (crosses) of $40 \mathrm{~dB}$. The measured bandwidth is $15.8 \mathrm{kHz}$. Fig. 6(b) presents the measured CMRR. The circled crosses are measurement results, the black line is the maximal CMRR of the op amp and the blue line is calculated with Eq. (2).

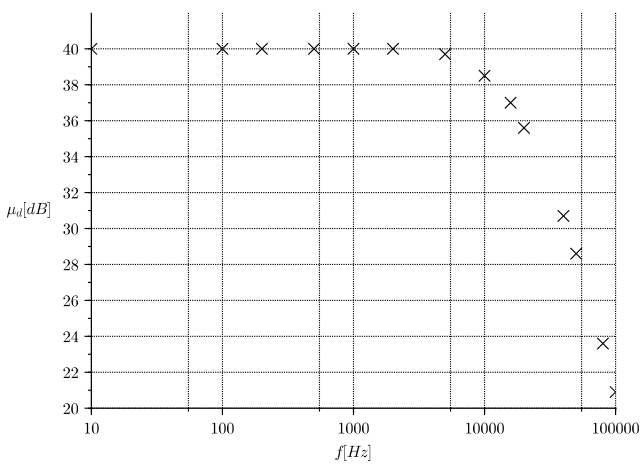

(a) $\mu_{d}$ as a function of frequency. All op amps: AD 822 .

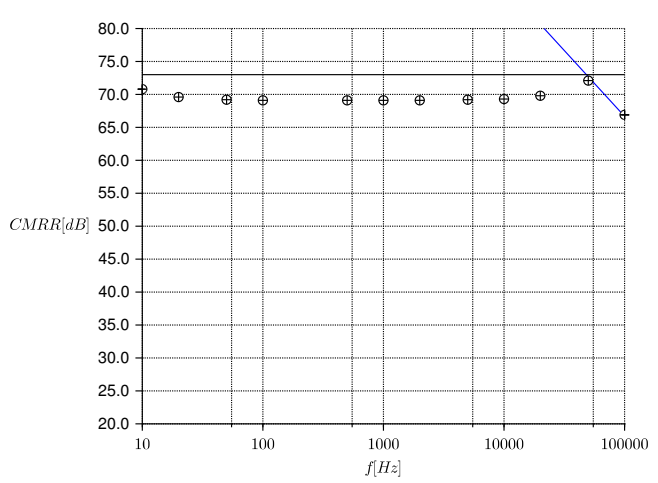

(b) CMRR as a function of frequency. All op amps: AD 822 .

Fig. 6: Differential voltage gain $\mu_{d}$ and CMRR of the IA. Black line: maximal low-frequency CMRR of the op amps. Blue line: calculated CMRR, Crosses and circled crosses: measurements.

Figs. 7(a) and (b) present the same measurements, but now op amp MCP 6002 is used in the transadmittance input stage. Since the MCP 6002 has a lower GBP (1 MHz typ.) than the AD822 (1.8 MHz typ) with this $R_{1}(10 \Omega)$, the bandwidth is $12.2 \mathrm{kHz}$. Both op amps are capable of low voltage operation, minimal supply voltage being $1.8 \mathrm{~V}$ and $\pm 2.5 \mathrm{~V}$, respectively.

It is demonstrative to see that the measured CMRR is large over a broader frequency range than that of the op amps themselves. The CMRR of the MCP 6002 is typically $77 \mathrm{~dB}$ at low frequencies, deteriorating to $20 \mathrm{~dB}$ at $10 \mathrm{kHz}$ and that of the $\mathrm{AD} 822$ is about $73 \mathrm{~dB}$ (low supply voltage) at 


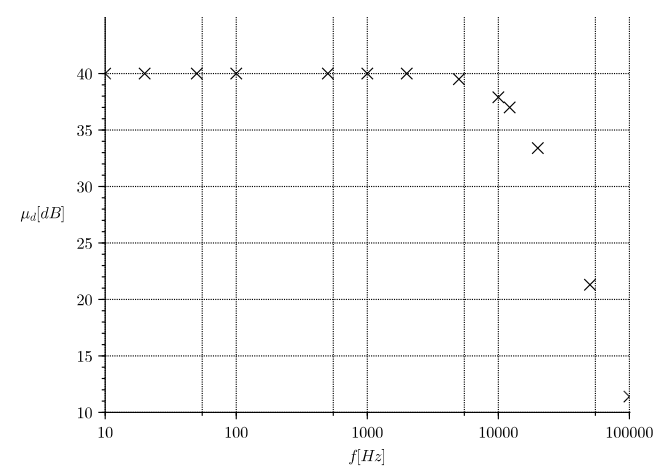

(a) $\mu_{d}$ as a function of frequency. Input stage op amps: MCP 6002, output stage op amps: AD 822.

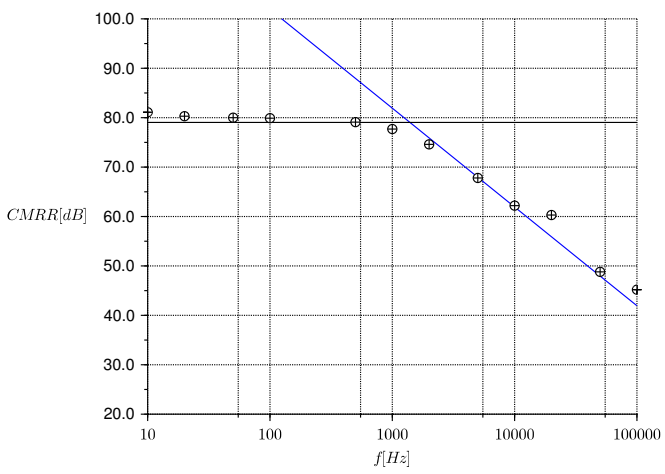

(b) CMRR as a function of frequency. Input stage op amps: MCP 6002, output stage op amps: AD 822.

Fig. 7: Differential voltage gain $\mu_{d}$ and CMRR of the IA. Black line: maximal low-frequency CMRR of the op amps. Blue line: calculated CMRR, Crosses and circled crosses: measurements.

low frequencies and deteriorating to ca. $40 \mathrm{~dB}$ at $100 \mathrm{kHz}$. The more constant CMRR up to $100 \mathrm{kHz}$ may be attributed to better matching and higher GBP of the AD 822 op amps.

\section{Discussion}

The IA presented here is capable of working at low supply voltages and shows a high CMRR over a broad frequency range, which makes it interesting in the present trend towards lower (system) supply voltages. On top of that, effects of mismatches between op amps can be evaluated using Eq. (2) enabling worst case analysis and design.

So, specifically in case of low-voltage applications and when the designer needs more design freedom to meet functional and EMC specifications, the IA presented in this paper may provide the intended combination of a reasonably high
CMRR up to $100 \mathrm{kHz}$, low noise behavior and being able to run on a low-voltage.

Apart from batteries, energy harvesting systems and DC-DC converters can also be used as floating power supplies, but this increases the risk of injecting extra interference to the voltage supply terminals of the op amps since DC-DC converters switch with a specific frequency.

\section{COnClusions}

This paper presents the design of an op amp based IA which CMRR does not depend on matching resistors and that is suitable for low-voltage applications. By selecting op amps with high gain bandwidth products and high (low-frequency) CMRR, the IA can reach high CMRR over broad frequency ranges. A rather typical rail-to-rail op amp (AD 822) is used in this design that still provides a CMRR greater than $60 \mathrm{~dB}$ at $100 \mathrm{kHz}$.

\section{REFERENCES}

[1] J. Xu, S. Mitra, C. Van Hoof, R. F. Yazicioglu, and K. A. A. Makinwa, "Active electrodes for wearable eeg acquisition: Review and electronics design methodology," IEEE Reviews in Biomedical Engineering, vol. 10, pp. 187-198, 2017.

[2] C. Keyer, F. Buesink, and F. Leferink, "Mains power synchronous conducted noise measurement in the 2 to $150 \mathrm{khz}$ band," in 2016 International Symposium on Electromagnetic Compatibility - EMC EUROPE, pp. 865$869,2016$.

[3] Z. Abidin, K. Tanno, S. Mago, and H. Tamura, "Low common-mode gain instrumentation amplifier architecture insensitive to resistor mismatches," International Journal of Electrical and Computer Engineering, vol. 6, no. 6, pp. 3247-3254, 2016.

[4] M. Mehrol, D. Goyal, and P. Varshney, "Differential voltage current conveyor transconductance amplifier based instrumentation amplifier," in 2016 IEEE 1st International Conference on Power Electronics, Intelligent Control and Energy Systems (ICPEICES), pp. 1-5, 2016.

[5] M. Konar, R. Sahu, and S. Kundu, "Improvement of the gain accuracy of the instrumentation amplifier using a very high gain operational amplifier," in 2019 Devices for Integrated Circuit (DevIC), pp. 408-412, 2019.

[6] I. M. Pandiev, "Design and implementation of difference and instrumentation amplifier's laboratory system for education in analog electronics," in 2020 XXIX International Scientific Conference Electronics (ET), pp. 1-4, 2020.

[7] M. A. P. Pertijs and W. J. Kindt, "A 140 db-cmrr currentfeedback instrumentation amplifier employing ping-pong auto-zeroing and chopping," IEEE Journal of Solid-State Circuits, vol. 45, no. 10, pp. 2044-2056, 2010.

[8] C. Kitchin and L. Count, "A designer's guide to instrumentation amplifiers," 3rd edition, Analog Devices, 2006.

[9] B. D. H. Tellegen, "On nullators and norators," IEEE transactions on circuit theory, pp. 466-469, Dec. 1966.

[10] J. H. Huijsing, Integrated Circuits for Accurate Linear Analogue Electric SIgnal Processing. PhD thesis, Delft University of Technology, 1981.

[11] "5.2 ti precision labs - op amps: Bandwidth - gain and gbw." https://training.ti.com/ ti-precision-labs-op-amps-bandwidth-gain-gbw, March 2015. 